Tohoku J. Exp. Med., 1990, 161, Suppl., 79-93

\title{
Two-Stage Model of Visual Pattern Discrimination Learning in Macaque Monkeys (Macaca mulatta and Macaca fuscata)
}

\author{
Eirchi Imai, Masao Yukie,* Joji Watanabe, ${ }^{*}$ Shigeya \\ Yaginuma, * Yasutaka Osawa* and Kazuo Hikosaka* \\ Division of Clinical Neurology and *Department of \\ Behavioral Pysiology, Tokyo Metropolitan Institute for \\ Neurosciences, Tokyo 183
}

Iwai, E., Yukie, M., Watanabe, J., Yaginuma, S., Osawa, Y. and Hikosaka, K. Two-Stage Model of Visual Pattern Discrimination Learning in Macaque Monkeys (Macaca mulatta and Macaca fuscata). Tohoku J. Exp. Med., 1990, 161, Suppl., 79-93 — To investigate the nature underlying the process of pattern discrimination learning, a series of seven experiments on seven working assumptions were undertaken. The main findings are as follows. The pattern discrimination learning consists of two time-dependent stages: The initial or first learning stage is the period of performance at chance, and the succeeding or second stage is the period of performance from just above the chance to a criterion level. The duration of the first stage is dependent on the degrees of cue-response separations, whereas that of the second stage is independent of. During the first stage, monkeys do not attend to the discriminative cue even at small cue-response separations, whereas during the second stage, they achieve pattern discrimination, or pattern perception and cognition, regardless of cue-response separations. After having learned the first pattern task with a cue-response separation, they learned new pattern tasks by means of the second stage, showing marked saving of the duration of the first stage. The findings in the present studies indicate that the first stage of learning is predominantly involved in the process of attending to the discriminative cues remote from the response site (a selective attention to the cue), whereas the second stage is concerned with the process of perception and cognition of the discriminative cue.— pattern discrimination; selective attention; acquisition learning; learning model ; monkeys

The role of selective attention to discriminative cues in learning has continued attract interest: The act of attending selectively to an object in the visual space (a selective attention) plays a critical role in the efficiency of its perception and cognition (cf. Parasuraman and Davis 1984). Several studies have provided substantial evidence for the mechanism of selective attention to the cue in discrimination learning. The influence of attention on learning depends on

For reprint: Dr. Eiichi Iwai, Division of Clinical Neurology, Tokyo Metropolitan Institute for Neurosciences, 2-6 Musashidai, Fuchu-City, Tokyo 183, Japan. 
tasks and testing procedures employed (Sutherland and Mackintosh 1971; Mackintosh 1983). An attention shift is intertwined with gaze shift (Yarbus 1976) and is possible to be achieved covertly by internal operations without overt eye and head movements (Posner 1980 ; Broadbent 1982). The attention shift to the cue, or how to attend to the cue, is a learned one (Zeaman and House 1963 ; Sutherland and Mackintosh 1971; Mackintosh 1983). The present study demonstrates that difficulty in the acquisition of pattern discrimination learning in monkeys is attributable largely to the difficulty in attending to discriminative pattern cues locating even at a very small distance from response site (the effect of small cue-response separations).

Aquisition scores of discriminations of patterns identical in configuration

Fig. 1 shows three pairs of plus sign and outline square patterns. At first sight, none may be aware of the differences of these three pairs. Quite accidentally, we noticed that monkeys learned these pairs with markedly different acquisition scores (the first working assumption). Also, it is frequently observed that the rate of acquisition of pattern discrimination by monkeys differs significantly from report to report, even though the pattern cues employed appear to be identical in configuration and the apparatus and testing procedures appear to be similar. Understanding such observations may provide insight into the mechanism of pattern discrimination. To confirm our inspection and to explain the nature underlying the above observations, we attempted and started the present studies about two decades ago, and are still doing further studies for this problem. Here, the outlines of the findings from the experiments for seven working assumptions will be described.
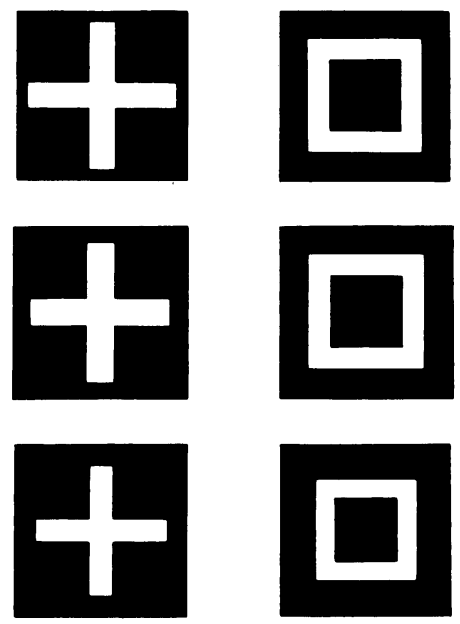

Fig. 1. Three pairs of pattern discriminanda identical in configuration. Top, Pair I; middle, Pair II ; bottom, Pair III. For explanation, see text. 


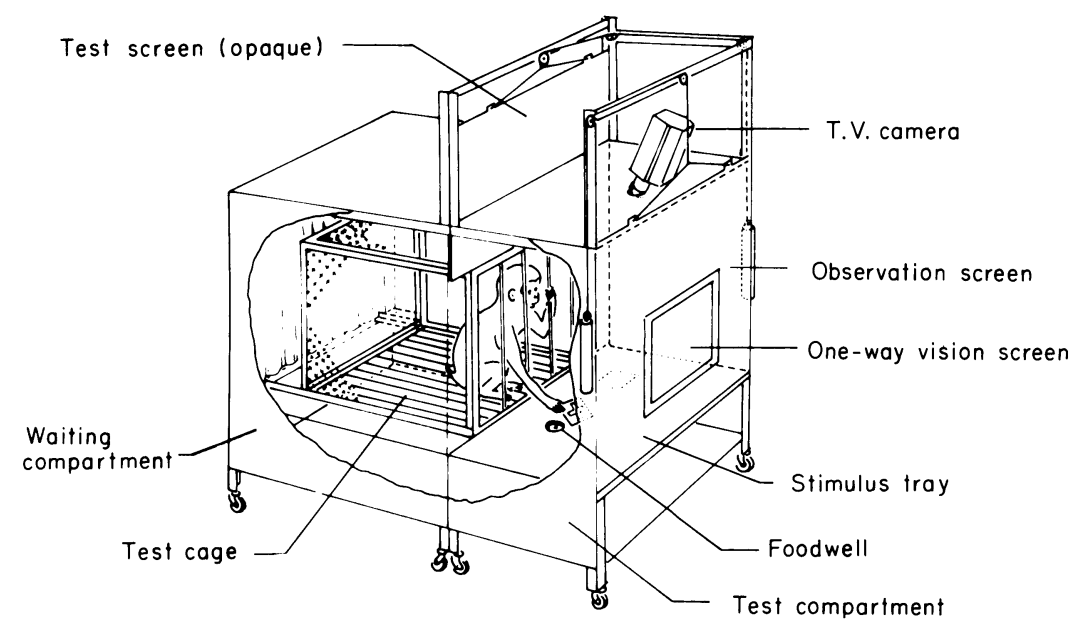

Fig. 2. Diagrammatical drawing of a modified WGTA (Wisconsin General Testing Apparatus) employed in our laboratory.

For pattern discrimination in monkeys studied in a modified Wisconsin General Testing Apparatus (WGTA; Fig. 2), pattern cues are displayed on background plaques (refer to Fig. 1), and monkeys touch the bottom corner or edge of a background plaque to push a stimulus card aside and to get a bait hidden under the plaque (refer to Fig. 2). They do not respond to the pattern cue directly (Butter and Gekoski 1966; Iwai and Mishkin 1968; Iwai et al. 1979). Accordingly, there is a small separation between the cue site and the response site to the bottom corner of a background plaque (cue-response separation). The drastic effect of spatial separations between the cue and response sites on learning is well-known (for reviews, Meyer et al. 1965; Stollnitz 1965; Cowey 1968). This concept has been established on the basis of detrimental effects from large separations more than $2.5 \mathrm{~cm}$ ( 1 inch). Although the effect of small separations less than $2 \mathrm{~cm}$ had not been studied, we assumed that the variance of the acquisition scores on patterns apparently identical in configuration would be attributable largely to the small differences in cue-response separations in the discriminanda (the second working assumption).

To confirm our observation mentioned above (the first working assumption), the acquisition scores on five pairs of pattern discriminanda identical in configuration in a WGTA by 223 experimentally naive macaque monkeys were compared each other. Seventy-five monkeys were trained on Pair I (Group I) ; 83 monkeys, on Pair II (Group II) ; 34 monkeys, on Pair III (Group III) ; 28 monkeys, on Pair IV (Group IV); and three monkeys, on Pair V (Group V). As seen in the illustrations in Fig. 3, each pair consisted of a white paper plus sign and an outline square displayed in the middle of dark gray cardboard plaques, but these pairs varied in either the size of the cue or the size of the background plaque. 


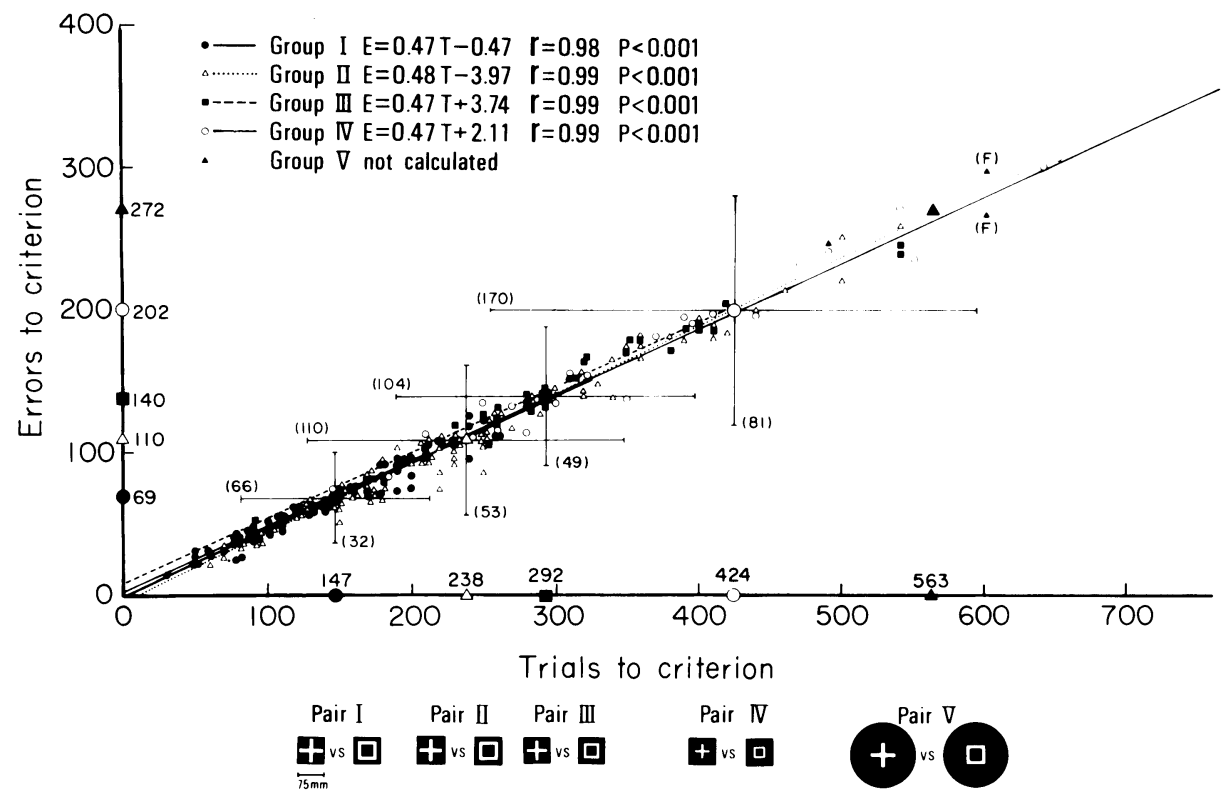

Fig. 3. Distribution of initial learning scores on five pairs with identical pattern cues in 223 experimentally naive monkeys. Individual learning scores are indicated with small symbols, and group means, with large ones: Trials are on abscissa, and errors, on ordinate. Thin lines crossing at large symbols denote standard errors (s.E.): Values in parentheses by horizontal lines are for trials, and those by vertical ones, for errors. Thick solid, dotted, dashed and thin solid lines denote regression lines of errors on trials for Groups I, II, III and IV, respectively: Formulas are at the left top. (F) by filled triangles for Group V indicates failure to attain criterion within the training limit of 600 trials. Illustrations in the botom are for Pairs employed in the present study.

For Pairs III (the pair shown in the bottom of Fig. 1) and IV, the pattern cues were proportionally reduced in size, seven-eighths and five-eighths, respectively, compared with the patterns of Pair I, and were centered on background of the same size as that used for Pair I (the pair in the top of Fig. 1). For Pair II (the pair in the middle of Fig. 1), the sizes of the pattern cues and the background plaque were slightly smaller and larger than those used for Pair I, respectively. For Pair V, the pattern cues were the same as those used for Pair I, but were displayed in the middle of enlarged cardboard disks. Therefore, the distance between the cue and the fringe of background plaque in Pair I was the smallest among the pairs, whereas that in Pair V was the largest. The white areas of the patterns of each member of a pair were the same. For each pair, the positive stimulus was the plus sign, response to which was rewarded with a raisin, while the negative one was the outline square, response to which was neither rewarded nor punished. The subjects were trained for 30 trials a day (a session of three blocks of 10 trials each) to a learning criterion of 90 or more correct choices of the positive 
stimulus in 10 consecutive blocks of 10 trials each.

The learning scores of the individual subjects and the group mean scores with five pairs of pattern discriminanda are plotted in Fig. 3. As seen, all subjects of Groups I, II, III and IV could learn Pairs I, II, III and IV, respectively. On the other hand, two subjects of Group V failed to learn Pair V within the training limit of 600 trials (Fig. 6). Although Pairs I to IV look very similar to the examiners (see the illustrations of Pairs in Fig. 3), the respective mean scores with Pairs II, III, IV and V were approximately one and a half, two, three, and four times the mean with Pair I. The differences between the group means were significant $(p s<0.01)$. Therefore, the results confirm our early observation on pattern discrimination, supporting our first assumption.

In addition, as seen in Fig. 3, there was a clear correlation between the trial and error scores for each group, even though the scores of individual subjects within a given group varied widely. The equations of the regression lines of errors on trials are given in the left top of Fig. 3. As seen, all correlation coefficient values were 0.98 or more. The four regression lines overlapped each other. In fact, the regression coefficients were homogeneous $(p>0.25)$. Therefore, the regression line of errors on trials through all 220 monkeys of Groups I to IV in the present study was found to be : $\mathrm{E}$ (errors) $=0.48 \mathrm{~T}$ (trials) -2.01 .

These findings indicate that monkeys learn the pattern tasks in a common manner in that they committed errors in about half of the training trials until attaining the learning criterion; they never randomly learn the pattern tasks.

\section{Learning scores as a function of cue-response separations}

The above finding suggested that learning scores depended on a common factor. In the present study, the sizes of the pattern cues and background plaques were varied experimentally. However, a size factor alone could not explain the present findings, because the sizes of the pattern cues of Pairs I and V and of the backgrounds of Pairs I, III and IV were exactly the same. In fact, any regression of learning scores on various size factors in the discriminanda could not be estimated as the best fitting one: Not only the linear components but also the nonlinear ones were significant ( $p \mathrm{~s}<0.05$; Figs. $4 \mathrm{C}$ to $4 \mathrm{E}$ ).

The above statistical results suggested that the learning scores must have regressed on a certain physical factor more fitly other than the size factors (the second assumption described before). Through several trend analyses, the best fitting linear regression of the learning scores was found on the logarithmic values of the distances from the pattern cues to the fringes of background plaques (Fig. $4 \mathrm{~A})$ : The linear component was significant $(p<0.01)$ and accounted for $99 \%$ of the variation due to the main effect, while the nonlinear one was not significant $(p>0.25)$.

As mentioned before, the distance from the cue to the fringe of the plaque can be taken as an index of the cue-response separation. The present finding indi- 

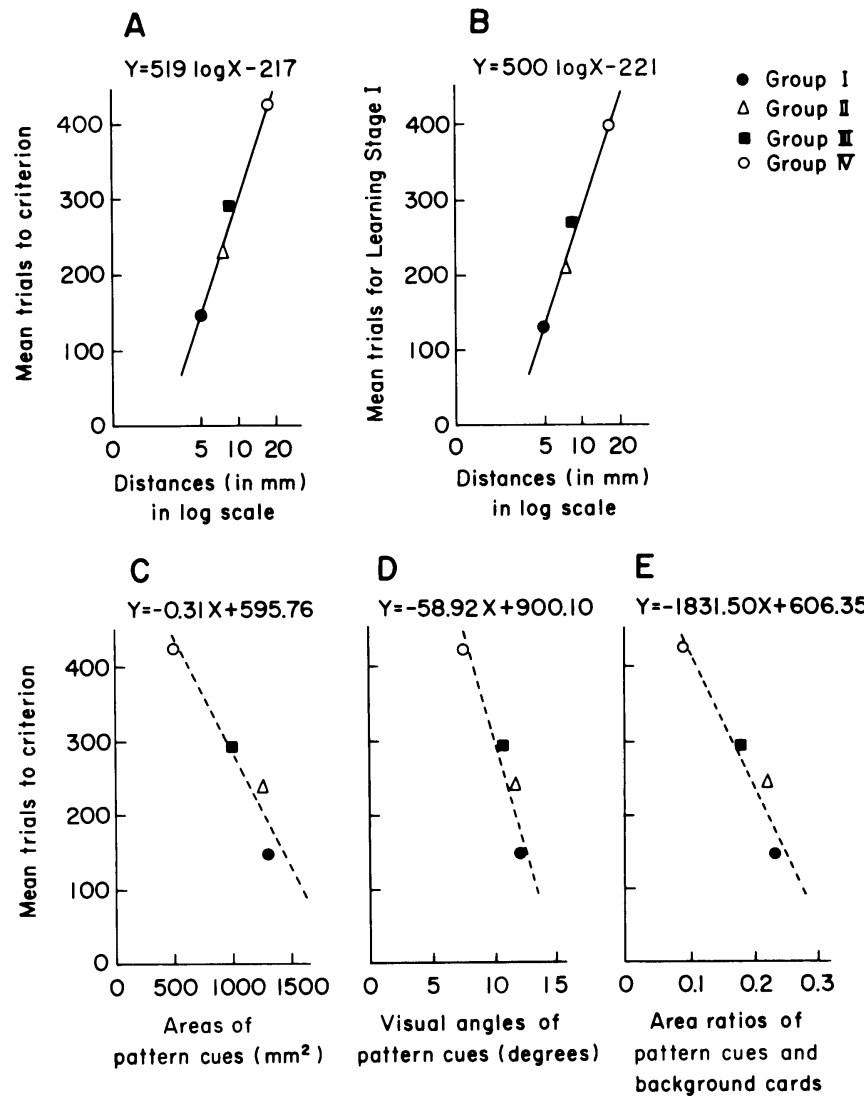

Fig. 4. Regressions of group mean learning scores on various physical factors in the discriminanda. A : Linear regression of group mean trials on logarithmic values of distances from bottoms of pattern cues to near fringes of background plaques. B : Linear regression of group mean trials for Learning Stage I on $\log$ distances. C : Regression of group means on values of areas of pattern cues. D : Regression of group means on values of visual angles of pattern cues. E : Regression of group means on values of area ratios of pattern cues to background plaques. Solid regression lines (A and B) denote that linear components are statistically significant $(p<0.01)$ and nonlinear ones are not $(p>0.05)$, whereas dashed lines (C, D and E) denote both components are significant $(p<0.05)$, showing any linear regression is not the best fitting one. Formulas for regression lines are presented at the top of each figure: Ys, learning scores on ordinates ; Xs, values of physical factors in the discriminanda on abscissae.

cates, therefore, that reducing the size of pattern cues and enlarging the size of background plaques increase the cue-response separations, and this significantly retards the efficiency of learning. The finding supports the second working assumption: The significant differences between the mean learning scores with pattern cues apparently identical in configuration are attributed to the effect of 
small cue-response separations.

The results from the subjects of Group V (Figs. 3 and 6) also supported this conclusion. Their results are shown in Fig. 6 by individual learning curves. As seen, their mean learning score was the largest among the group means and the distance from the cue to the fringe of background plaque (or, the degree of the cue-response separation) was the largest among the pairs.

\section{Learning curves on pattern pairs}

The finding that ratios of errors to trials were almost the same across the groups suggested that regardless of the discriminanda employed, the subjects attained learning abruptly following initial performance at the chance level (the third working assumption). To examine this supposition, the analysis of learning curves on Pairs I, II, III and IV was made.

Fig. 5 indicates the backward learning curves on the four pairs, I to IV, according to the method of Hayes (1953 ; cf. Iwai 1971). As seen, the curves can be described by two clearly different stages: The stage of initial performance at the chance level and the succeeding abrupt stage of improvement from the chance to the criterion level. More important, the four curves overlapped each other:

\section{Backward learning curves}

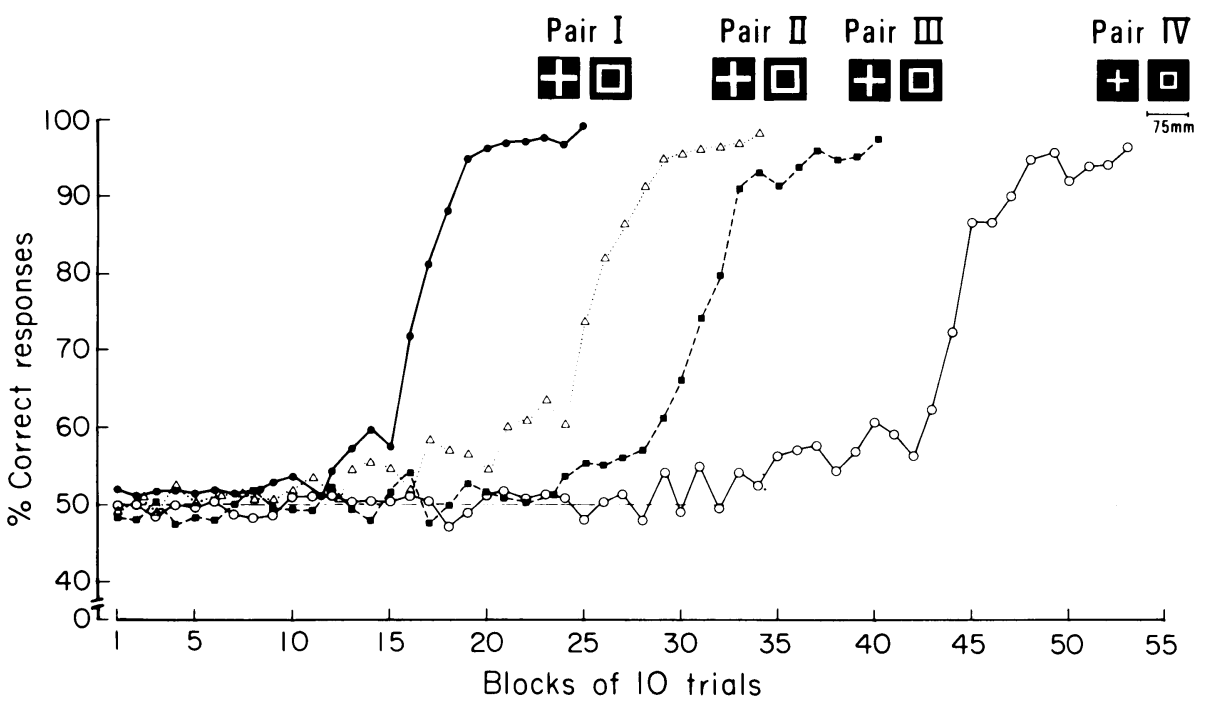

Fig. 5. Averaged backward learning curves of pattern discriminations on Pairs I, II, III and IV. Curves are plotted according to the method of Hayes (1953). Group mean percentages of correct responses in each block are plotted from the final block of the criterion trials backward group mean blocks: Scores of subjects having reached learning criterion with fewer test blocks than the group mean blocks were assigned with their first block scores for subsequent blocks to maintain the number per group throughout. 
The only differences between them were the durations of the initial stage.

To confirm the validity of this inspection on the curves statistically, the performance at the chance $(p>0.05)$ was defined as the level with $19(64 \%)$ or fewer correct responses in 30 trials of three consecutive blocks and was labeled Learning Stage $I$, and that above the chance $(p<0.05)$ was defined as the level with $20(67 \%)$ or more correct responses in 30 trials and was labeled Learning Stage II. The group differences between the mean scores for Learning Stage I were significant $(p \mathrm{~s}<0.01)$, whereas the differences between the means for Learning Stage II were not $(p s>0.05)$.

The results supported the third assumption. The finding indicates that the process of pattern discrimination learning consists of, at least, two separable learning stages.

The results from the subjects of Group V supported this conclusion. As seen in Fig. 6, the learning curve of one monkey who could learn Pair V documented the same feature as those of the backward averaged curves of Groups I to IV ; the initial long duration of Learning Stage I and the succeeding short and sharp period of Learning Stage II. On the other hand, the other two monkeys remained at the chance level performance (Learning Stage I) even after the training limit of 600 or more trials.

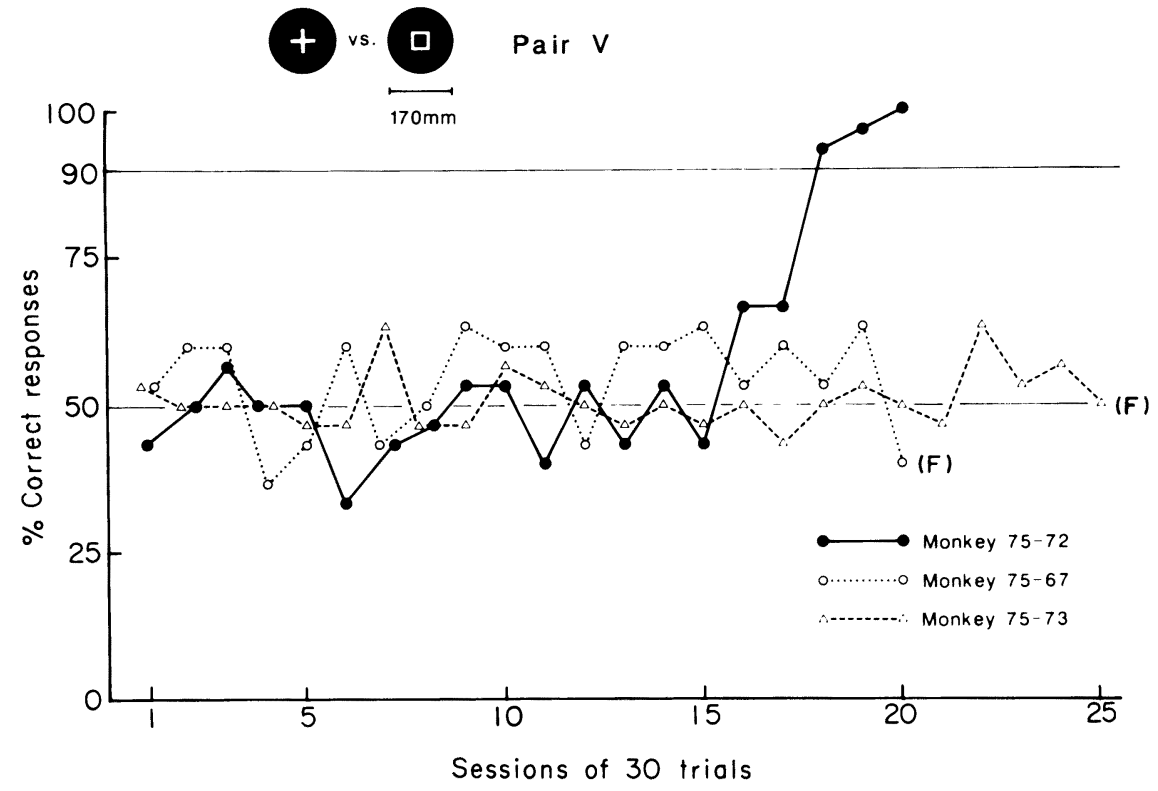

Fig. 6. Learning curves of individual subjects of Group V on Pair V. Notice that the characteristic of learning curve of one monkey succeeding in learning on this pair is the same as those of the curves in Figure 5, and that two other subjects remained at chance level (Learning Stage I) even after 600 or more trials. 
Scores for learning stage-I as a function of cue-response separations

The above findings suggested that these two stages, Learning Stages I and II, would represent different mechanisms in pattern discrimination learning, respectively. Therefore, the following four experiments were undertaken to investigate the nature underlying the two stages in pattern discrimination learning.

The fourth working assumption was that the finding of the linear regression of the acquisition learning scores on the distances between the cue and response site would be attributable to the linear regression of the scores for Learning Stage I on the distances and not to those for Learning Stage II, because the scores for Learning Stage II were almost the same across the group means.

The results are indicated in Fig. 4B. The scores for Learning Stage I regressed linearly on the logarithmic values of the distances from the pattern cues to the fringes of background plaques: The linear component was significant $(p<0.01)$ and accounted for $99 \%$ of the variation due to the main effect, and the nonlinear component was not significant $(p>0.25)$.

The results supported the fourth assumption. The finding indicates that the regression of the learning scores on cue-response distances (Fig. 4A) is dependent exclusively on the scores for Learning Stage I (Fig. 4B) and was independent of the scores for Learning Stage II. It is concluded, therefore, that even a small increment of cue-response separations results in remarkable prolongation of Learning Stage I, but does not affect Learning Stage II.

\section{Effect of prior training during learning stage-I on succeeding learning}

The above finding suggested that during Learning Stage I monkeys might be ignorant of the discriminative cues, whereas they might be aware of and sample the cues during Learning Stage II. Therefore, the fifth working assumption was that when training on a first task with a cue-response separation was discontinued during Learning Stage I, this training experience would not significantly influence learning a second task. We expected this results even when the cues of the second task were identical in configuration to those of the first task.

To examine this assumption, four naive monkeys of Group VI were first trained on Pair IV, but training was discontinued after 360 trials (12 session trials). Then, they were trained to learn Pair I. The results are shown in Fig. 7A. For Pair IV, they remained at the chance level of performance throughout the training period (Left panel of Fig. 7A): The mean percentage of correct responses during the final 100 trials was $48 \%$ correct. The result on Pair I is shown by the backward learning curve in the middle of Fig. 7A, together with that of 75 naive monkeys of Group I with Pair I (from Fig. 5). As seen, both learning curves of Groups VI and I overlapped each other: In fact, there was no significant difference between the group means $(p>0.10)$. The results showed that the scores for Learning Stages I and II of the subjects of Group VI on Pair 
A
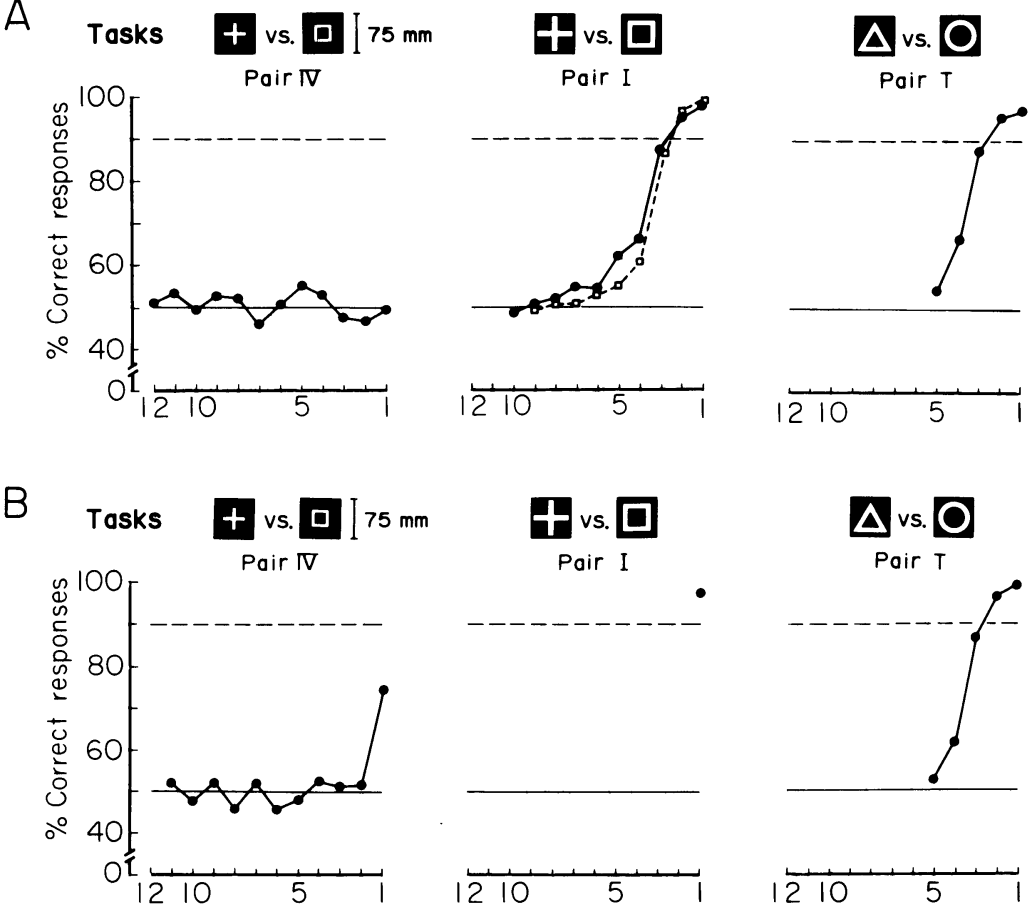

Sessions of 30 trials

Fig. 7. Averaged backward learning curves of discriminations on Pairs I and $\mathrm{T}$ at respective test phases. Pairs used in tests are illustrated above the curves. A is for Group VI, and B, for Group VII. Curve with open circles and dashed line shown in middle panel of Figure $\mathrm{A}$ is acquisition curve of 75 naive monkeys of Group I with Pair I (from Fig. 5). Notice that for pretraining shown in left panels, subjects of Group VI were trained on Pair IV for 360 training trials and their performance remained at the chance level, whereas subjects of Group VII were trained on Pair IV until reaching performance level of $67 \%$ or a few more correct responses. Also, notice that as seen in middle panel of B, subjects of Group VII were tested on Pair I for only a session of 30 trials ; because, unexpectedly, all subjects showed 9 or 10 correct responses even in the first test block of 10 trials.

I did not deviate from the normal range of naive monkeys.

The results supported the fifth assumption. The finding indicates that prior training experience during Learning Stage I does not influence the succeeding learning: monkeys learn the second task, as if they were experimentally naive.

\section{Effect of prior training until learning stage-II on succeeding learning}

In contrast to the fifth working assumption, the sixth one was that when pretraining on the first task was discontinued at a performance level a little better than the chance (the beginning period of Learning Stage II), monkeys would learn 
the second task quickly: Because, as mentioned, it would be assumed that at this time, they would have been aware of and started sampling the discriminative cues of the first task.

As studied for the fifth assumption, four naive monkeys of Group VII were first trained on Pair IV : However, in this experiment, they were trained until reaching a performance level of $20(67 \%)$ or a few more correct responses in three consecutive blocks of 10 trials each. Then, they were trained on Pair I. The results are shown in Fig. 7B. For Fair IV, they reached the level of $67 \%$ correct with the mean of 328 trials (left panel of Fig. 7B). For Pair I, unexpectedly, all subjects attained the criterion without requiring any additional training trials (middle panel of Fig. 7B) : They immediately performed on the second task, Pair I, as if they had learned it before.

The results supported the six assumption. The finding indicates that the training on the first task to the level a little better than the chance was significantly effective in learning the second pattern task. Therefore, the difference between the findings with Pair I for the fifth and sixth assumptions is attributed to the difference in the preceding training levels on Pair IV. These findings strongly support the conclusion that cue-response separations affect exclusively Learning Stage I, but does not affect Learning Stage II.

\section{Pattern discrimination learning after attaining pattern discrimination}

The above findings suggested that after learning a pattern task with cueresponse separation, monkeys would learn a new pattern task with maked saving of the duration of Learning Stage I (the seventh working assumption).

All subjects of Groups VI and VII were trained on a new pattern task, Pair $\mathrm{T}$, consisting of an outline triangle and an outline circle (for this pair, refer to the illustrations in Fig. 7). The results are shown in the right panels of Fig. 7A and 7B, respectively. All learned Pair T rapidly. As seen, both curves overlapped each other $(p>0.25)$, and also that of Group VI with Pair I and those of Groups I, II, III and IV with Pairs I, II, III and IV, respectively, in the acquisition study (Fig. 5) : The only differences between them were the durations of Learning Stage I. The mean scores for Learning Stage I of both groups, VI and VII, with Pair T were remarkably smaller than that of Group VI with Pair I, and also than those in the acquisition learning on Pairs I, II, III and IV $(p s<0.01)$. On the other hand, both means for Learning Stage II of Groups VI and VII with Pair T did not differ from any mean for Learning Stage II with Pairs I to IV $(p s>0.25)$.

The results supported the seventh assumption. There was a possibility, however, that Pair T might be a particularly easy task to learn. If so, the above finding with Pair T would be meaningless. To examine this possibility, six naive monkeys of Group VIII were first trained to learn Pair T and then to learn Pair I. The results from this group with Pairs $\mathrm{T}$ and I were entirely the same as those from Groups VI and VII with Pairs I and T, and thus supported the findings for 
the sixth assumption with Pair T.

These findings indicate that regardless of patterns, monkeys learn new pattern tasks with marked saving of the duration of Learning Stage I after learning the first pattern task with a small cue-response separation. Therefore, these findings for the fifth to seventh assumptions indicate that the effect of cue-response separations can be learned and transferred across the problems (Mackintosh 1983).

\section{Consideration on processes underlying pattern discrimination learning}

Effect of small cue-response separations on learning. As mentioned before, the concept of the effect of cue-respose separations on learning has been established on the drastic effects from large separations: The effect from small ones has not been demonstrated yet. It is found in the present studies that even an increment of separation as small as about $0.3 \mathrm{~cm}$ results in a marked retardation of the acquisition of pattern discriminations. For example, the respective mean scores of Groups I and II were 147 (69) and 238 trials (110 errors). Therefore, the effect of cue-response separations is significant across small separations as well as large ones.

As to the nature underlying this effect, early, Schuck (1960) and Polidora and Fletcher (1964) proposed an attention hypothesis that cue-response separations might affect the act of attending to the cues remote from the response site. However, no studies have provided supporting evidence for this assumption yet. The present studies provide several findings for the nature underlying the difficulty in pattern discrimination learning at small cue-response separation (1) The separation affects only Learning Stage I and does not influence Learning Stage II. (2) The larger the separation, the longer was the duration of Learning Stage I. (3) The duration of Learning Stage I is a function of the degrees of the separations, (4) More important, mokeys are ignorant of the cue even at small cue-response separations during the initial long period of Learning Stage I. (5) The effect of cue-response separations on learning depends on prior learning experience. These findings indicate that even a small increment of cue-respose separation in discriminanda makes it significantly difficult for mokeys to attend selectively to the discriminative cue (act of selective attention to the cues).

The concept of the effect of cue-response separations is established mainly on the basis of the findings in macaque monkeys. The effect has been confirmed in human (Nazzaro et al. 1971) and squirrel monkeys (Abordo and Lee 1977) On the other hand, there are few studies for this effect in nonprimates (Milner et al. 1979, for rats; Riley and Roitblat 1978, for pigeons). As the present study shows, the effect of cue-response separations, even small ones, can not be neglected in understanding the mechanism of discrimination learning. The act of attending to the relevant cue even at small cue-response separations should be taken into account when studying learning in many animals.

Role of selective attention to cues in discrimination learning. Naive mokeys 
require many training trials to attain pattern discrimination learning (refer to Fig. 5). However, the present studies demonstrate that pattern discrimination by itself is easily attained : Apparent difficulty in the acquisition learning is attributable largely to the difficulty in attending to pattern cues even at small cueresponse separations. In addition, it is demonstrated that the observation that the acquisition learning scores on patterns identical in configuration differ significantly from report to report is explainable by small differences in cueresponse separations among the discriminanda. Furthermore, the present findings indicate that the act of attending to cues at cue-response separations can be learned and transferred across problems (Zeaman and House 1963; Sutherland and Mackintosh 1971; Mackintosh 1975, 1983). These findings indicate that the act of selective attention to cue plays a critical role in learning, as recent cognitive psychology has emphasized.

As Schuck (1960) and Polidora and Fletcher (1964) suggested, the present studies indicate that during the initial large period of Learning Stage I, monkeys focus their attention on the background plaque (or, the response site and its neighboring parts); they do not attend to the pattern cues. To achieve pattern discrimination, therefore, they must disengage their attention from the response site and shift their attention to the relevant cue. The finding that the duration of Learning Stage I in a function of distances between the cue and response sites suggests that such an attention shift is performed gradually along the way from the response site toward the relevant cue even at a small cue-response separation: Such a slow shift of the attention may be made covertly without overt eye and head movements (Posner 1980 ; Braoadbent 1982; Kurtz et al. 1982 ; Posner et al. 1984).

On the other hand, it is demonstrated in the present studies that discriminative pattern stimuli by themselves are perceived and cognized during Learning Stage II after attending selectively to the cues. It is well-known that inferotemporal cortex of macaque monkeys is involved modality-specifically and closely in visual pattern perception and cognition (cf. Iwai 1980, 1982, 1985 ; Iwai and Mishkin 1968; Iwai et al. 1979). Many inferotemporal neurons respond differentially to various pattern stimuli (Iwai 1980, 1982, 1985; Iwai et al. 1987). Whereas no neurons respond to stimuli other than those of visual modality (Gross 1973). Recently, it was found that about $30 \%$ of inferotemporal neurons became responsive to a warning auditory stimulus given before the presentation of patterns (Iwai 1985; Hikosaka et al. 1986). The warning stimulus given in the learning paradigm may put monkeys in readiness for attending to coming pattern stimuli. The present behavioral findings and the above electrophysiological finding as well indicate, therefore, that the act of focusing attention on the cues markedly improves the efficiency of discrimination learning on the cues.

It is assumed that there are at least two time-dependent components underlying the act of attending to the cue (a selective attention) in visual discrimina- 
tion learning. The first component is the process of disengaging the current attention from the irrelevant site or target and shifting attention to the relevant cue or object, and this process is achieved during Learning Stage I defined in the present study. The second component is the process of focusing attention on the relevant cue to achieve perception efficiently, and this process is maintained throughout Learning Stage II.

Two-stage model of learning. It is demonstrated that pattern discrimination learning consists of two time-dependent stages, the initial Learning Stage I and the succeeding Learning Stage II, and that the respective stages are involved in the different mechanisms of learning. The finding is in part consistent with an earlier two-stage model of learning surmised from the observation for retarded children (Zeaman and House 1963). The present studies provide enough evidence for understanding the mechanism of visual learning and thus extend the domain of learning theory. As mentioned above, Learning Stage $I$ is involved in the process of attending to the relevant cues. On the other hand, the process of Learning Stage II is concerned with the process of perceiving and cognizing the cues per se with the keep of focusing attention selectively to the cues.

\section{References}

1) Abordo, E.J. \& Lee, J. (1977) Effect of introducing novel configulational cues on a learned color discrimination in squirrel monkeys. Percept. Mot. Skills, 44, 147-151.

2) Broadbent, D.E. (1982) Task combination and selective intake of information. Acta Psychol. (Amst.), 50, 253-290.

3) Butter, C.M. \& Gekoski, W. (1966) Alterations in pattern equivalence following inferotemporal and lateral striate lesions in rhesus monkeys. J. Comp. Physiol. Psychol., 61, 309-312.

4) Cowey, A. (1968) Discrimination. In: Analysis of Behavioral Change, edited by L. Weiskranz, Harper \& Row, New York, pp.189-238.

5) Gross, C.G. (1973) Visual functions of inferotemporal cortex. In: Handbook of Sensory Physiology, Vol VIII/3, Central Visual Information, Part B, edited by R. Jung, Springer-Verlag, Berlin, pp. 451-482.

6) Hayes, K.J. (1953) The backward curve: A method for the study of learning. Psychol. Rev., 60, 269-275.

7) Hikosaka, K., Watanabe, J., Sato, T., Suyama, H. \& Iwai, E. (1986) Responsiveness to patterns in inferotemporal neurons in monkeys performing discrimination task. $J$. Physiol. Soc. Jpn., 48, 275.

8) Iwai, E. (1971) A backward relearning curve by unilaterally eye-enucleated gold fish. Fukushima J. Med. Sci., 18, 29-34.

9) Iwai, E. (1980) Visual mechanisms in the temporal and prestriate association cortices of the monkey. In : Brain and Behavior, edited by G. Adam, I. Meszaros \& E.I. Banyai, Pergamon Press, New York, pp. 279-286.

10) Iwai, E. (1982) A model regarding system of information processing in visual goal-directed behavior of macaque monkeys. In : Learning and Memory, edited by S. Saito \& J.L. McGaugh, Excerpta Medica, Amsterdam, pp. 39-58.

11) Iwai, E. (1985) Neurophysiological basis of pattern vision in macaque monkeys. Vision Res., 25, 425-439.

12) Iwai, E. \& Mishkin, M. (1968) Two visual foci in the temporal lobe of monleys. In: 
Neurophysiological Basis of Learning and Behavior, edited by N. Yoshii \& N.A. Bushwald, Osaka Univ. Press, Osaka, pp. 23-33.

13) Iwai, E., Osawa, Y. \& Utsumi, Y. (1979) Elevation of visual pattern discrimination limen in monkeys with total removal of inferotemporal cortex. Jpn. J. Physiol., 29, 749-765.

14) Iwai, E., Aihara, T. \& Hikosaka, K. (1987) Inferotemporal neurons of the monkey responsive to auditory signal. Brain Res., 410, 121-124.

15) Kurtz, D., Leiby, C.C., III \& Butter, C.M. (1982) Further analysis of S-R separation effects on visual discrimination performance of normal rhesus monkeys and monkeys with superior colliculus lesions. J. Comp. Physiol. Psychol., 96, 35-46.

16) Mackintosh, N.J. (1975) A theory of attention: Variation in associability of stimuli with reinforcement. Psychol. Rev., 82, 276-279.

17) Mackintosh, N.J. (1983) Conditioning and Associative Learning. Claredon Press, Oxford.

18) Meyer, D.R., Treichler, F.R. \& Meyer, P.B. (1965) Discrete-trial training techniques and stimulus variables. In: Behavior of Nonhuman Primates, Vol. 1, edited by A.M. Schrier, H.F. Harlow \& F. Stollnitz, Academic Press, New York, pp. 1-49.

19) Milner, A.D., Goolale, M.A. \& Morton, M.C. (1979) Visual sampling after lesions of the superior colliculus in rats. J. Comp. Physiol. Psychol., 93, 1015-1023.

20) Nazzaro, J.N., Rodrigues, R. \& Nazzaro, J.R. (1971) Configulation variables in visual discrimination leaning. J. Genet. Psychol., 85, 45-50.

21) Parasuraman, R. \& Davis, D.R. (1984) Varieties of Attention, Academic Press, New York.

22) Polidora, V.J. \& Fletcher, H.J. (1964) An analysis of the importance of S-R spatial contiguity for proficient primate discrimination performance. J. Comp. Physiol. Psychol., 57, 224-230.

23) Posner, M.I. (1980) Orienting of attention. Q.J. Exp. Psychol., 32, 3-25.

24) Posner, M.I., Walker, J.A., Friedrich, F.J. \& Rafal, R.D. (1984) Effects of parietal injury on covert orienting of attention. $J$. Neurosci., 4, 1865-1874.

25) Riley, D.A. \& Roitblat, H.L. (1978) Selective attention and related cognitive processes in pigions. In : Cognitive Processes in Animal Behavior, edited by S.H. Hulse, H. Fowler \& W.K. Honing, Erlbaum, Hillsdale, N.J., pp. 249-276.

26) Schuck, J.R. (1960) Pattern discrimination and visual sampling by the monkey. $J$. Comp. Physiol. Psychol., 53, 251-255.

27) Stollnitz, F. (1965) Spatial variables, observing responses, and discrimination learning sets. Psychol. Rev., 72, 247-261.

28) Sutherland, N.S. \& Mackintosh, N.J. (1971) Mechanism of Animal Discrimination Learning, Academic Press, New York.

29) Yarbus, A.L. (1976) Eye Movements and Vision, Plenum Press, New York.

30) Zeaman, D. \& House, B.J. (1963) The role of attention in retardate discrimination learing. In: Handbook of Mental Deficiency, edited by N.R. Ellis, McGraw-Hill, New York, pp. 159-223. 